Birgit R. Erdle

\title{
Der phantasmatische und der decouvrierte weibliche Körper
}

\author{
Zwei Paradigmen der Kulturation
}

Wenn wir nach der Rolle des Körpers in Kultur- und Geschlechter-Theorien fragen, sehen wir uns sogleich in eine Zweideutigkeit verwickelt. Diese entsteht daraus, daß der Körper im philosophischen und im anthropologischen Diskurs in doppelter Weise eingesetzt wird. Einerseits nämlich wird er als physischer Raum der Erfahrung präsentiert, als »Lagerstätte und Träger der Empfindnisse«, wie Emmanuel Lévinas unter Bezug auf die Phänomenologie Husserls schreibt (Lévinas 1983, 177), andererseits hingegen als - anatomisch, biologisch, ethnologisch, künstlerisch - betrachteter und abgezeichneter Leib, als Bild unter Bildern. Der Körper gehört so gleichzeitig zwei voneinander geschiedenen Ordnungen an: einmal der Ordnung des Symbolischen - denn er ist von jeher mit Sprache überzogen und durch sprachliche Konzepte historisch konstruiert -, zum anderen der außerhalb des Zeichens liegenden Ordnung des Realen, da sich in ihm Lust, Genuß, Schmerz, Traumata, Zeitlichkeit und Tod abzeichnen. Die Sphäre des Leiblichen deutet so auf eine andere, in der abendländischen Philosophiegeschichte vergessene oder geleugnete Grundbedingung menschlicher Existenz, die Lévinas als unreines Sein kennzeichnet - ein Sein, dessen SelbstgewiBheit durchkreuzt, verunreinigt, verletzt wird durch das Andere der Sprache, des Sexuellen, des Schmerzes, des Schocks und des Todes. Lévinas erklärt daher, die Leiblichkeit stelle »die ontologische Verfassung einer primären Selbstentfremdung « (Lévinas 1987, 329) dar. Doch werde im Leib »das sFürsich der Person nicht nur verkannt, sondern mißhandelt, nicht nur verletzt, sondern gezwungen. 'Ich bin alles, was Sie wollen, sagt Sganarelle unter den Schlägen. «(Lévinas 1987, 334)

\section{Körper/Zeichen: Zwei Versionen der Verknüpfung}

Die beiden Texte von Gertrud Kolmar und Sigmund Freud, auf die ich mich im folgenden beziehe und die beide weiter unten im Wortlaut abgedruckt sind, präsentieren dargestellte weibliche Körper, die dazu dienen, eine komplexe, teilweise widersprüchliche Deutung jenes reziproken Geschehens zu entwerfen, welches ich mit dem Begriff Kulturation bezeichne. In beiden Texten wird ein Extrempunkt, eine kritische Wendung, vielleicht auch eine Pointe der Kulturation offengelegt - 
ein Stadium des Zusammenbruchs oder der völligen Erstarrung. Es scheint mir dabei kein Zufall, daß beide Paradigmen der Kulturation, die ich aufzuzeichnen versuche, sich auf eine Darstellung weiblicher Körper gründen. Denn das Weibliche bietet sich nicht nur als bevorzugte, leere Fläche der kulturellen Inskription an, wie etwa Marina Warner aufzeigt (1985) - es dient nicht nur als On der Verhandlung kultureller und gesellschaftlicher Normen, es erlaubt nicht nur das "gendering" eines kulturellen oder hermeneutischen Konfliktes (wie wir im Falle von Freuds Text sehen werden). Der dargestellte weibliche Körper scheint auch in besonderer und »unheimlicher« Weise jene Stelle zu bezeichnen, an der sich eine Verschränkung von Realem und Bildhaftem zeigt.

Nun sind die Präsentationsweisen des weiblichen Körpers, über welchen sich die Texte Kolmars und Freuds entfalten, deutlich voneinander unterschieden. In Kolmars Gedicht wird der weibliche Körper als ein phantasmatischer präsentiert, während bei Freud ein Corpus von Texten - ein jüdischer Witz und zwei einander durchkreuzende, kommentierende Passagen des Autors, die in dessen Reflexionsvorgang eingebettet sind - einen weiblichen Körper verhüllt, der sich als decouvrierter darbietet. Den Begriff der Präsentation wähle ich, um anzudeuten, daß es sich um einen dargestellten wie auch angeschauten Körper handelt - und beide Texte ziehen uns als Lesende in dieses beinahe voyeuristische, Lust, Abscheu oder Empathie erweckende Zuschauen mit hinein.

Die beiden Paradigmen der Kulturation, die ich skizzieren möchte, beziehen sich auf den jeweils verschiedenen Modus, in dem die somatische Einzeichnung der dominanten Kultur bei Kolmar und Freud erscheint, das heißt, auf die Verknüpfung, die sich in einer bestimmten sozialen, existentiellen und bildlichen Konstellation zwischen soma und sema herstellt. Kolmars Text, der sich aus der psychotischen Rede eines weiblichen Subjekts heraus bildet, zeigt diese Verknüpfung zwischen soma und sema als einen Einbruch der Dimension des Bildes, der Darstellung, in die Sphäre des Leiblichen - einen Einbruch, der dem Moment des Schocks und der Demütigung für das Subjekt, das ihn erleidet, Dauer verleiht. Den sich hier ereignenden Kollaps der Differenzen - und die gleichzeitige Realisierung einer eindeutigen, ontologisch (oder religiös) hegründeten Unterscheidung zwischen Mensch und Nichtmensch - versuche ich mit Hilfe des totalitären Paradigmas zu beschreiben. Der Text Freuds, anhand dessen ich das Paradigma der Assimilation entwerfen möchte, handelt von der semiotischen Entkleidung eines weiblichen Körpers: eine unter extremen Schmerzen gebärende Frau durchquert die Schichtungen jener (väterlichen) Sprachen, die sie sich angeeignet hat, um sich der herrschenden Kultur zu assimilieren, an ihrer Bildung und der Feinheit ihrer Sitten teilzuhaben. Auch wenn die semiotische Entblößung den weiblichen Körper in seiner sprachlichen Nacktheit preisgibt (denn die gebärende Frau findet sich reduziert auf ihre Muttersprache, welche ihrerseits zusammenschrumpft zu einem Schrei), bleibt der decouvrierte weibliche Körper - im Unterschied zum phantasmatischen - im Raum des Intersubjektiven und der sprachlichen Differenz.

In beiden Texten geht es auch, wie wir sehen werden, um die Konstruktion eines das (welches?) Selbst beruhigenden Anderen: doch während diese Andersheit bei Freud zuerst sexuell definiert wird (der weibliche Körper wandelt sich zum müt- 
terlichen), konfrontiert uns Kolmar mit einer Definitionsregel, die sich aus einer Verschmelzung von medizinischen, christlichen, strafrechtlichen und mythischen Kriterien herleitet. Die Kategorie der sexuellen Differenz verschwindet in dieser Definitionsregel - das Andere wird als hassenswertes, abstoßendes Scheusal vorgestellt.

\section{Sigmund Freud: Der einäugige Witz}

Als exemplarisches poetisches Bild fuir das erste Paradigma der Kulturation, das ich skizzieren möchte, das der Assimilation, ${ }^{1}$ lese ich die Anekdote von der vornehmen gebärenden Frau, auf die Sigmund Freud in seiner frühen Studie Der Witz und seine Beziehung zum Unbewußten eingeht. Sie lautet:

Der Arzt, der gebeten worden ist, der Frau Baronin bei ihrer Entbindung beizustehen, erklärt den Moment für noch nicht gekommen und schlägt dem Baron unterdes eine Kartenpartie im Nebenzimmer vor. Nach einer Weile dringt der Wehruf der Frau Baronin an das Ohr der beiden Männer. "Ah mon Dieu, que je souffre! « Der Gemahl springt auf, aber der Arzt wehrt ab: "Es ist nichts, spielen wir weiter. "Eine. Weile später hört man die Kreißende wieder: „Mein Gott, mein Gott, was für Schmerzen!" - „Wollen Sie nicht hineingehen, Herr Professor? " fragt der Baron. - „Nein, nein, es ist noch nicht Zeit." - Endlich hört man aus dem Nebenzimmer ein unverkennbares: "Ai, waih, waih" geschrien; da wirft der Arzt die Karten weg und sagt: "Es ist Zeit." (Freud 1982, 78)

Die Anekdote taucht in Freuds Text an zwei verschiedenen Stellen auf - und dieser doppelte Auftritt erklärt sich aus Freuds Zögern, ob die Geschichte der Kategorie des Witzes oder jener des Komischen zuzuordnen sei. Zuerst bestimmt er die Anekdote als guten Witz, der sich dazu eigne, die Technik der »Darstellung durch ein Kleines « zu veranschaulichen. Diese Darstellingsweise besteht darin, wie Freud erklärt, einen ganzen Charakter durch ein winziges Detail zum Ausdruck zu bringen - sie fordert also eine symbolisierende Lesart, welche die Anekdote als rhetorische Figur des pars pro toto auffaßt. Daraus ergibt sich folgende erste Deutung:

Wie der Schmerz durch alle Schichtungen der Erziehung die ursprüngliche Natur durchbrechen läßt, und wie eine wichtige Entscheidung mit Recht von einer scheinbar belanglosen Äußerung abhängig gemacht wird, das zeigt beides dieser gute Witz an dem Beispiel der schrittweisen Veränderung der Klagerufe bei der gebärenden vornehmen Frau. (Freud 1982, 78)

Diese erste Kommentierung Freuds zeigt deutlich die Bemühung um eine Universalisierung des Sinns, in dem die weibliche (mütterliche) Figur, der jiddische Klageschrei und der jüdische Kontext der Geschichte ausgespart bleiben. In ihrer Untersuchung zu Freuds Witz-Buch argumentiert Sarah Kofman daher, Freud leugne im Zeichen eines universellen Humanismus gleichzeitig die »Kastration« 


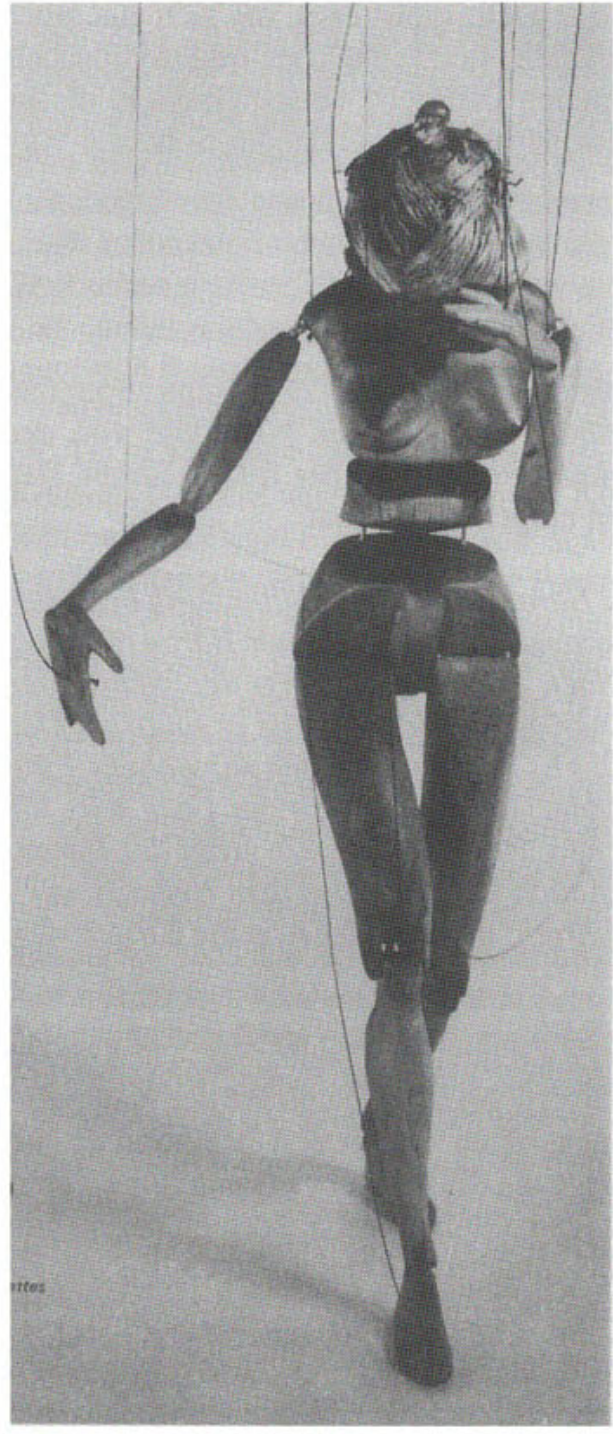

W. A. Dwiggins (1880-1956), Die Marionette Lilith

(Aus: Fragments for a History of the Human Body I, Zone. New York 1989)

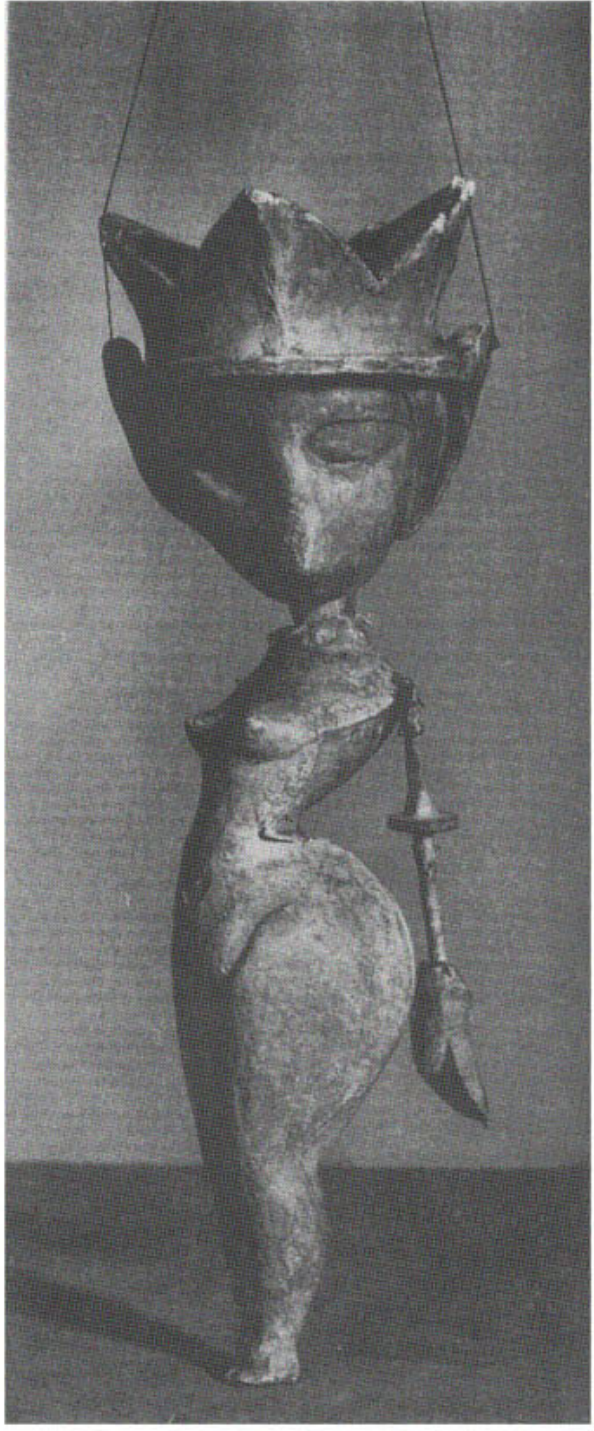

Michael Carr (1907-28), Marionette einer nackten Königin.

(Aus: Fragments for a History of the Human Body I, Zone. New York 1989) 
des Juden und die "Kastration « der Frau zugunsten des Konzepts eines universellen Menschseins: »Wir sind alle Menschenkinder « (Kofman 1990, 27).

Doch gerade am Topos des weiblichen, des mütterlichen Körpers kehren beide »Beschneidungen «, beide »Mängel « zurück. Und dieser Wiederkehr, die Freud bemerkt, begegnet er mit einer zweiten, revidierten Deutung der Anekdote: Nun behandelt er sie nicht mehr als Witz, sondern als Beispiel einer komischen Entlarvung, welche auf die Herabsetzung des Erhabenen zielt. Die Entlarvung sei, so erläutert Freud, dort angezeigt, »wo jemand Würde und Autorität durch einen Trug an sich gerissen hat, die ihm in der Wirklichkeit abgenommen werden müssen." (Freud 1982, 187)

Bevor ich auf die drei Kernbegriffe dieser zweiten Deutung Freuds, welche die Anekdote als conte morale liest, eingehe - nämlich jemand, Trug und in der Wirklichkeit -, möchte ich das Konzept des Komischen in Freuds Theorie kurz erläutern, um die doppelte Herabsetzung und Demaskierung verständlich zu machen, die der Kategorienwechsel vom Witz zum Komischen impliziert - eine Herabsetzung und Demaskierung, die sich, wie Sarah Kofman schreibt, ebenso auf die Frau wie auf die Anekdote beziehen (Kofman 1990, 156). Wenn Freud den Witz als Januskopf charakterisiert, der gleichzeitig zwei Herren dient, indem er zugleich an zwei unterschiedlichen psychischen Orten - dem BewuBtsein und dem Unbewußten - spielt, so erscheint ihm das Komische als Verkümmerung (Freud 1982, 200) des Witzes: nämlich als ein Januskopf, dessen eines Gesicht leer ist, der beschnitten ist und daher dem Weiblichen ähnelt. Aufgrund dieser Struktur läßt er die Suche nach einem tieferen Sinn ins Leere laufen, treibt die Deutung stets zu der einen Hälfte des Doppelgesichts, zur Fassade zurück - zur Fassade des BewuBten, des Textuellen und des Weiblichen. "Man gerät auf nichts, wenn man sich von der Technik ins UnbewuBte verlocken läßt«, stellt Freud fest (Freud 1982, 200).

Im Zuge der Herabsetzung des Witzes zur komischen Anekdote wandelt sich, so erläutert Sarah Kofman, das Objekt der Entlarvung: nicht mehr die ursprüngliche menschliche Natur wird nun entblöBt, wie noch in der ersten Deutung, sondern die Natur der Frau, die in dem unbestirnmten Pronomen jemand verschwindet - so wie das personale Pronomen je in den tiefer liegenden Sprachen verschwindet. Die sexuelle Differenzierung, die Freuds zweite Deutung einführt, würde dann der Erniedrigung der Mutter dienen, dem Lachen über sie; sie würde erlauben, die ehrbare, reine, gebildete Mutter zu entkleiden, um in Augenschein zu nehmen, was sich unter ihren vornehmen Kleidern verbirgt: eine einfache Frau, deren Schreie keine Anzeichen ihrer Bildung und der Verfeinerung ihrer Sitten mehr zeigen.

Indessen spricht aber die komische Anekdote von einer doppelten Entblößung - einer sexuellen und einer kulturellen. In diesem Sinne würde das Wort Trug, das den Angelpunkt in Freuds zweiter Deutung bildet, sich auf die jüdische Herkunft beziehen, auf die erste, ins UnbewuBte eingesenkte Sprache, die unter der Verkleidung der vornehmen Sprachen hervorbricht. Denn Trug bedeutet ja nicht nur Betrug - jene Begabung zur Verstellung und Schauspielerei, die mit der Natur des Weiblichen verknüpft wird -, sondern auch Sinnestäuschung. In dieser Lesart würden wir dann dem begegnen, was ich eingangs als "gendering « einer kulturellen Ambivalenz bezeichnet habe: das Eingeständnis, wie zerbrechlich, wie leicht zu erschüttern die Fassade der Assimilation ist, kann sich nur in der Einschränkung 
der sexuellen Differenz äußern - in der Bindung der verräterischen Muttersprache an den mütterlichen Körper.

In der Anekdote erscheint der weibliche Körper als Sprachmedium: Erst wenn die gebärende Frau die im Laufe ihres Lebens gelernten, aufeinandergeschichteten Sprachen durchquert hat, erst wenn sie sich ihrer täuschenden vornehmen Kleidung im Zuge ihrer semiotischen Enthüllung entledigt hat, wird ihr vom Arzt und vom Ehemann Trost und helfender Beistand gewährt. Die Anekdote scheint so das Vorhandensein einer ersten, quasi natürlichen, mütterlichen Sprache zu implizieren, der gegenüber die zweiten, väterlichen Sprachen als Maskierungen, Verkleidungen, Rollen gewertet werden - als Dissimulation, welche (im Gegensatz zur Simulation) fingiert, etwas, das man hat, nicht zu haben. Diese Maskierungen, die das Realitätsprinzip unangetastet lassen, ermöglichen eine Assimilation an die herrschende Kultur, lagern die in der Verschiedenheit der Sprachen tätige Differenz im Selbst ab und bergen die Schutzlosigkeit des Leibes. Die Decouvrage der Sprachschichten steuert dagegen auf jenen Moment des Authentischen zu, in dem sich eine transparente Beziehung zwischen dem Körper und dessen sprachlicher Äußerung herstellt - ein Augenblick tödlicher Bedrohung. Wir können daher von einer nicht nur kulturellen, sondern auch semiotischen EntblöBung sprechen. Im Zuge dieser Entkleidung anerkennt die gebärende Frau ihre Herkunft als existentielles Datum.

Der letzte Klageruf in der ersten Sprache markiert jenen Moment, in dem die symbolische Struktur der Sprache zerbricht und übergeht in eine Interjektion, einen Aufschrei. Unter dem extremen physischen Schmerz, den die gebärende Frau erleidet, löst sich ihre vertraute Welt auf, wie Elaine Scarry schreibt: »Der Schmerz hat die Macht, das Subjekt und die Zeichen der Wirklichkeit zu vernichten « (Scarry 1990, 15). Durch den Schmerz und das Ausbleiben der Hilfe wird sie zu einer EntblöBung gezwungen, die der Logik eines Geständnisses entspricht, wie der narrative Verlauf der Anekdote der Logik eines Paroxysmus folgt. Nun wird deutlicher, was Freuds Wendung in der Wirklichkeit bedeuten könnte: einen Ort oder Moment des Entzugs vertrauter Wirklichkeit, eine Erfahrung blendenden Schmerzes, welche die Vorstellung von Kontinuität zerreiBt, einen Augenblick tödlicher Bedrohung, der dem nahezukommen scheint, was Lacan als das Reale bezeichnet, vor welchem die symbolische Ordnung versagt.

Der Körper der gebärenden Frau ist dem männlichen Zuschauer auf mehrfache Weise preisgegeben, denn er richtet sich an vier männliche Adressaten - den Arzt, den Ehemann, den Analytiker Freud und den impliziten Leser. Der Arzt, der den Ehemann in das Bündnis gegen die Frau hineinzieht, um sein Kartenspiel nicht zu früh unterbrechen zu müssen, entziffert die Sprachlaute als somatisches Symptom, das ihm für eine korrekte Indikation ausreicht. Im Handeln des Arztes offenbart sich eine grundlegende Ambiguität: entweder er durchschaut den Betrug, vielleicht auch die Selbstverkennung der gebärenden Frau, weil er die Fähigkeit besitzt, die topischen Differenzen (Kofman 1990, 160), die verschiedenen Tiefendimensionen zwischen den drei Sprachen zu hören. Oder er führt der Frau durch seine Indifferenz vor Augen, daß es ihr nicht gelingen kann, ihren Schmerz in der Sprache der assimilierten Kultur zum Ausdruck zu bringen. Die Komplizität mit der herrschenden Kultur würde dann für die Frau bedeuten, daß sie ihre Bedrängnis und ihren physischen Schmerz zwar in dieser Kultur darstellen kann, daß aber diese Darstel- 
lung übergangen wird, sie also in der Wirklichkeit der dominierenden Kultur unsichtbar oder unhörbar bleibt.

Von einer zweiten grundlegenden Ambiguität ist der dritte Klageruf der Frau geprägt, welcher den Bruch der symbolischen Ordnung wie auch die momentane Rücknahme der Assimilation ankündigt: indem er den Endpunkt jener Durchquerung der übereinander geschichteten Sprachen markiert, die von der Zurückhaltung des Arztes erzwungen wird, bringt er eine Aufgabe des Reichtums an sprachlichen Differenzen zum Ausdruck, die sich über eine stufenweise Entfaltung vollzieht, um im gleichen Zuge eine leibliche Geschlechterdifferenz zu konstituieren.

Und die lachenden Dritten? Mit seiner zweiten Deutung der Anekdote, welche die hermeneutische Geste der Universalisierung zurücknimmt, anerkennt Freud die Beunruhigung, welche von der Fragilität der Fassade der Assimilation in der Wirklichkeit ausgelöst wird. Im selben Zuge jedoch leugnet er diese Destabilisierung, da er sie auf das Feld der sexuellen Differenz verschiebt und so zu einem geschlechtsspezifischen Ereignis erklärt. Auch die lachenden Dritten werden von dieser Beunruhigung erfaßt. Da sich aber sowohl die Decouvrierung, das die-Fassung-verlieren, wie auch der Schmerz ganz in die Figur des Weiblichen, genauer: des Mütterlichen, zurückgezogen hat, können sie die doppelte Entblößung der Frau genießen und ihren eigenen Trug verdecken. Doch gerade der Körper der gebärenden vornehmen Frau, so argumentiert Sarah Kofman, lehre Freud, zwischen den verschiedenen Schichten der Sprachäußerung zu unterscheiden, das heißt, von der Position des Ehemanns, für den alle drei Sprachen dasselbe anzeigen, zur Position des Arztes überzuwechseln, der die topischen Differenzen innerhalb des Gesprochenen berücksichtigt und sich selbst dadurch gleichzeitig als Archäologe und als Übersetzer konstituiert. In Freuds zweiter Deutung der Anekdote kündigt sich dies an, wenn das Faktum des Schmerzes verborgen wird in der topischen Umschreibung in der Wirklichkeit, während die in der ersten Deutung hervorgehobene ursprüngliche Natur, die alle Schichtungen der Erziehung durchbricht, sich gewandelt hat zur Aufdeckung eines Trugs - einer Aufdeckung, die an die Durchquerung der Sprachschichten gebunden ist und mit dieser einhergeht. So läßt die Bewegung von der ersten zur zweiten Deutung, die der Reflexionsvorgang Freuds vollzieht, auch erkennen, wie Freud sein theoretisches Projekt auf dem weiblichen Sprachkörper gründet. 


\title{
3. Gertrud Kolmar: Figur einer Verwerfung
}

\author{
Die Irre
}

Beaune, Côte d'Or, den 14.10.1927

Mit runzligen Lippen schlüre ich Wermut.

Von meinen Nüstern tropft RuB und Teer.

Meine Augen liegen auf Feldern, bestellt mit Scbwermut,

Und darum habe ich keine Blicke noch Tränen mehr.

Mein Kind wohnt ganz allein

Im Garten unter dem harten, mächtigen Stein.

$O$ seht! O seht! Welch einen Kopf muß ich tragen!

Rot und gelb, halb Schwefel, halb Ton.

Der meine ward mir zetbrochen und abgeschlagen

Vom Fallbeil der Großen Revolution.

Da hat mich der Böse durch alle. Stementiere,

Lowen und Widder, gehetz

Und mir im Krebs den Kopf einer Teufelin aufgesetzt.

Jäger und Schergen, Henkersknechte,

O Gendarmen der ganzen Welt in Wut!

Mein häBliches Haupt tut doch nicbt das Schlechte;

Schaut ber! Meine Hände sind gut.

So schön mit Blumen geschmückt wie ein Grab, als sein Grab.

Ich pflückte sie alle den Parkbeeten und den Kränzen am Totenmal ab.

Ich will alles Land erfüllen mit meinen lauten Gladiolen,

Mein Herz zerreiß ich in Nelken, es über den Erdball zu streun,

Über ganz Frankreich, über ganz Deutschland, über ganz Belgien. über ganz Polen!

Für meinen Sohn soll das sein; da wird er sich freun.

Er kam aus dem Kriege mit einem zu wilden, zottigen Bart,

Und sie fürchteten sich vor ihm und haben ihn eingescharrt.

Die Stadt wächst immer größer, je weiter ich gebe,

Sie reckt sich, verrückt sich, daB ich mein Ziel nie erreichen mag.

Wenn ich abends am Friedbofstor stehe,

Kehrt es sich von mir fort, jedesmal, in den morgigen Tag.

Ich setz mich vors Schulhaus, nicke den Kleinen mit meinem roten Krebshaupt voll Grind;

Denn wo ich auch sitze: immer geh ich zu meinem Kind.

(Kolmar 1960, 90)

Gertrud Kolmar wurde 1894 in Berlin geboren, in eine assimilierte großbürgerliche Rechtsanwaltsfamilie, die sich in der deutschen Geschichte und Kultur verwurzelt fühlte. Ihre Schwester Hilde Wenzel berichtet: »Als einzige aus der Familie war sie schon als junges Mädchen dem zionistischen Gedanken zugänglich und lehnte die 
Einflüsse der wilhelminischen Epoche bewuBt ab«(Hilde Wenzel, in: Kolmar 1960, 596). Ihr glückte die Emigration nicht; sie blieb bei ihrem Vater in Berlin, durchlebte alle Phasen der Verfolgung, bis sie vermutlich im März 1943 nach Auschwitz deportiert wurde. Zuvor hatte sie begonnen, Hebräisch zu lernen und in hebräischer Sprache Gedichte zu schreiben, die offenbar verlorengegangen sind.

Das zitierte Gedicht findet sich im »Dritten Raum« des Zyklus »Weibliches Bildnis«, der in vier "Räume " unterteilt ist. Wir können vermuten, daß der Text autobiographisch geprägt ist - in jenem Sinn, daB sich in ihm der Gestus der Selbst-Identifizierung ausprägt. ${ }^{2}$ Im Gewand der Rollenlyrik spricht ein weibliches Ich, das von Panik befallen ist - es ist aufgelöst, de-subjektiviert, hat sich zu einem monströsen, abstoßenden Wesen gewandelt. Der Text ist gereizt, affiziert durch eine Psychose, in der die Grenzen zwischen dem Symbolischen und dem Imaginären völlig zusammengebrochen sind. Da das Ich in der Psychose aus der symbolischen Ordnung, welche die zwischenmenschliche Realität strukturiert, ausgestoßen wird in eine isolierte, losgelöste Rede, können wir die Referenzpunkte, die "objektive « grammatische Ordnung dieses in der Ich-Form vorgetragenen Monologs nicht festmachen - wir werden bei der Lektüre des Textes ganz in den dissoziativen Sog der psychotischen Rede hineingezogen. Die Grenzlinie, die es ermöglichen würde, zwischen den »inneren «, auf die psychotische Symptomatik hindeutenden Bildern und jenen (»äußeren«) Bildern, die von der umgebenden, dominanten Kultur entworfen und auf den Körper des weiblichen Subjekts projiziert wurden, zu unterscheiden, ist vom Text ausgewischt.

Bezieht sich also der psychotische Effekt, der sich an der Stelle des weiblichen Körpers zeigt, auf psychische Mechanismen der sozialen Umgebung oder auf solche des sprechenden Ich? Läßt sich der Körper oder die Rede des Ich als Symptom der dominanten Kultur selbst lesen, als ein Symptom, das auf ein Projektionsgeschehen, auf Mechanismen der Verleugnung (der Realität einer traumatisierenden, das Selbst ursprünglich verwundenden und beschneidenden Wahrnehmung) und der Verwerfung (eines fundamentalen Signifikanten) im Inneren der herrschenden Kultur selbst deutet? Jacques Lacan hat in seiner Diskussion des Begriffs der Verwerfung darauf hingewiesen, daß die verworfenen Signifikanten (im Unterschied zur Verdrängung) nicht in das UnbewuBte des Subjekts integriert werden und daher nicht aus dessen »Inneren « wiederkehren, sondern mitten im Realen erscheinen, besonders im halluzinatorischen Phänomen (Laplanche/Pontalis 1989,608$)$.

Läßt sich daher das Schreckbild, das Kolmars Text entwirft, als eine solche Wiederkehr entziffern? Der Text weigert sich, diese Frage zu lösen: vielmehr läßt er sie als Frage bestehen, als sei er selbst von Panik durchdrungen. Er wiederholt damit jene Bewegung, in der sich das Ineinanderstürzen der imaginären und der symbolischen Ordnung, der Zusammenbruch vertrauter Konventionen und Zivilisationsregeln abzeichnet.

Wie aber ist nun der weibliche Körper in diesem Text dargestellt? Wir können zwei Aspekte jener Gewalt unterscheiden, die sich an der Stelle des weiblichen Körpers materialisiert: Einmal geht es um eine Enthauptung und Behauptung, einen Akt der Substitution, der einer Idolisierung ähnelt - einer Frau wird der Kopf abgeschlagen und an dessen Stelle der »Kopf einer Teufelin« aufgesetzt. Zum 
zweiten geht es um einen Akt der Verkörperung oder der Kondensation: denn es entsteht eine neue leibliche Ganzheit; in der für den Betrachter (oder für den Verfolger) Körper und Abbildung verschmelzen.

Für eine Erläuterung des ersten Aspekts scheint es nützlich, die Diskussion des Antlitzes bei Emmanuel Lévinas hinzuzuziehen. In der philosophischen Ethik von LÉvinas nimmt das menschliche Antlitz die Stelle ein, an der die Spur des Anderen (l'autre) erscheint - die Spur jenes Unendlichen jenseits des Seins, die sich nicht thematisieren läßt. Doch teilt sie sich leibhaftig und unmetaphorisch mit im ethischen Verhältnis zum anderen Menschen ( $l^{\prime}$ autrui), im »face à face « des Blicks in sein Antlitz (le visage). Die lebendige Gegenwart des Antlitzes, so erläutert Lévinas, »empfängt ihre Ordnung von der Abwesenheit, in der das Unendliche sich nähert; von ihrem Un-Ort. Sie hat ihre Ordnung in der Spur ihres eigenen Fortgangs" (Lévinas 1983, 284). Um die Bewegung des jenseits des Seins anzudeuten, spricht Lévinas von der Notwendigkeit, "sich ab-zusetzen; einen Akt der Absetzung zu vollziehen, in dem Sinne wie man von abgesetzten Königen spricht. Diese Ab-setzung der Souveränität durch das Ich ist die soziale Beziehung zum Anderen « (Lévinas 1986, 39) - der Begriff des Sozialen scheint hier dem des Intersubjektiven oder des Sprachlichen sehr nahezukommen. Die Verantwortung für den Anderen beschneidet das Selbst - es handelt sich also um eine Beziehung, die nicht symmetrisch ist. Um sich vor einem idyllischen Verständnis des Begriffs des Anderen bei Lévinas zu hüten, und um das Denken jener anderen Struktur der Subjektivität zu nähern, die seine Philosophie beschreibt, ist es hilfreich, daran zu erinnern, wie die Wirksamkeit des Anderen sich entfaltet - nämlich darin, daß sie die Kontinuität des Selben durchbricht, indem sie einhergeht mit einer Verletzung, einem Einbruch oder einer Anklage.

Legt man diesen theoretischen Entwurf des Anderen, der auch die Denkbewegung selbst immer wieder ins Leere führt, zugrunde, so läßt sich die Umwendung des Anderen in das Selbe als Umwendung eines nicht identifizierbaren Anderen in ein identifizierbares Anderes verstehen. Der Logik einer solchen Umwendung, die auf eine Identifizierung des Anderen zielt, da das Nicht-identifizieren-können eine zu große und dauernde Erschütterung im Selben auslöst, scheint jener Akt der Substitution zu folgen, der in Kolmars Gedicht dargestellt ist. Diese Gewalttat ist gleichsam mehr oder anderes als Mord, da sie ja nicht in der Enthauptung endet, sondern die Behauptung ausführt, das Böse sei nun in physischer Gestalt identifizierbar. Es handelt sich hier um eine Symbolisierung in einem doppelten Sinn: das weibliche Ich ist symbolisch fixiert - denn das Teufelsbild repräsentiert das Böse schlechthin und verweist auf eine jahrhundertealte religiöse Bildtradition -, gleichzeitig aber symbolisch $t o t$ - ortlos, sozial ausgeschlossen, verfolgt von der Wut der Ordnungshüter. Die Tilgung des Anderen, die in der Substitution des Kopfes dargestellt wird, artikuliert sich als Externalisierung: das Böse, der Schrecken, wird vom Inneren des Selbst auf ein davon getrenntes Außen übertragen und darin fixiert, indem es einen von diesem Selbst unterschiedenen, anderen Körper erhält. Die Darstellung, die Kolmar wählt, zeigt, wie gerade durch die Konstruktion und die Repräsentation des Anderen Andersheit abgewehrt, identifiziert und fixiert wird.

Wir könnten diesen Zusammenhang weiter erläutern, indem wir einige Überlegungen von Slavoj Zizek aufgreifen, der das psychoanalytische Konzept des 
(großen und kleinen) Anderen von Lacan für eine Analyse des ideologischen Diskurses nutzt. Zizek weist auf eine Ambivalenz in der Präsentation des Anderen durch den ideologischen Diskurs hin: Einerseits garantiert dieser Sinn, indem er ein konsistentes Deutungsmuster anbietet - wie etwa die Figur des Juden im Antisemitismus (Zizek 1989, 176). Doch zugleich ist der Andere auch der Ort reinen Scheins - und um seine Nicht-Existenz, das Faktum, daß er immer schon tot ist, zu verbergen, ist es notwendig, seine Reinheit unbedingt zu wahren und ihn daher vor jeder heterogenen Geste zu beschützen (Zizek 1991, 84).

Ich möchte aber diese Überlegungen hier nicht fortfürren, sondern mich nun dem zweiten Aspekt jener Gewalt zuwenden, die sich an der Stelle des weiblichen Körpers in Kolmars Text zeigt: dem Verschwinden der Unterscheidung zwischen der Sphäre des Leiblichen und der Dimension des Bildes - genauer gesagt, dem Impuls, diese Unterscheidung unkenntlich zu machen, der sich dem weiblichen Körper in Kolmars Text einprägt, ihn spaltet. Das sprechende Ich verkörpert das Bild des Bösen - als hätte Kolmar diesen Satz wörtlich genommen, um dessen buchstäblichen Sinn, den realen Kern, den sie in ihm spürte, freizulegen. Indem das sprechende Ich des Textes durch die Verkörperung die ihm gewaltsam übergestülpte Abbildung zum Leben erweckt, bekräftigt es gerade jene Ordnung, die es peinigt, die es dehumanisiert, in die Verbannung und in den symbolischen Tod treibt. Die Beziehung zwischen soma und sema, die diese neu produzierte leibliche Ganzheit kennzeichnet, deutet nicht nur auf einen mimetischen Impuls, sondern vielmehr auf eine Fusion und eine Konfusion zwischen Körper und Bild, zwischen dem figurativen und dem buchstäblichen Sinn einer Aussage oder einer Drohung. Dies impliziert eine Verletzung des Realitätsprinzips (im Sinne von Freud) und im selben Zuge ein Unlesbar-machen der Tilgung des Anderen.

Was Kolmars Text hier inszeniert, ist der fatale, gewaltsame Akt einer Verkörperung und einer Verdichtung, ins Buchstäbliche und Leibhaftige gewendet: die imaginäre Produktion einer unwiderruflichen Identität von Körper und stereotyper Abbildung. Anders ausgedrückt: die rhetorische Struktur der Verdichtung (verschiedener Signifikanten, wie etwa des Bösen, des Häßlichen) und die Konfusion der Sphäre des Zeichens mit jener des Realen - beides strukturelle Merkmale, die im Antisemitismus wirksam sind - werden anhand eines weiblichen Körpers vorgeführt. Die durch diese Operationen entstandene Figur ist inszeniert einzig und allein zum Zweck der Sichtbarkeit: sie erfüllt so die Intention eines Blicks, der die Möglichkeit unterbindet, daß sich das Angeschaute entzieht oder verhüllt, und der dem Erblickten die paradoxe Logik aufzwingt, als Anderes zugleich völlig ausgelöscht und total sichtbar zu sein. Lévinas bezeichnet dieses Starren mit dem Wort dé-visager, das ein Entkleiden, ein Sich-entledigen des Antlitzes meint und auf diese Weise die identifizierende Geste unterstreicht, die diesem begierigen Sehen-wollen innewohnt.

Das Schwinden der Grenzen zwischen Abbildung und Körper - und die dadurch hervorgerufene Auflösung der materiellen Realität im Imaginären - bestimmt den Symptomcharakter der beschriebenen Figur: in der Terminologie Lacans markiert das Symptom genau den Kreuzungspunkt, in dem die Ordnungen des Realen, des Symbolischen und des Imaginären einander überlagern. Zizek schlägt eine Analogic vor zwischen dem Symptom und der Rolle, die das Lacansche Andere im 
ideologischen Diskurs spielt: beide gewähren dem Subjekt Konsistenz, beide stabilisieren das Selbst.

Wenn die in Kolmars Text dargestellte weibliche Figur, die das Böse im wörtlichen Sinne und auf unheimliche Weise verkörpert und veranschaulicht, als Symptom oder als objet $a$ aufgefaBt werden kann, so bedeutet dies, daB sie sich als Effekt jener Dynamik im psychischen Apparat beschreiben läBt, welche die Wirksamkeit des Unheimlichen - die Destrukturierung des Ich - zu unterbinden sucht, indem sie ihm ein Objekt gibt und es dadurch externalisiert. Wie ich schon andeutete, kommt dieser Vorgang dem sehr nahe, was Lacan als Verwerfung beschreibt. Im Antisemitismus geschieht dies, wenn die Figur des imaginären Juden identifiziert wird mit dem Tod und mit jenen Momenten der Moderne, die auf den Tod deuten: Momenten der Krise, des Exzesses, der Entwurzelung.

Doch in welcher Weise können wir bei der im Gedicht Kolmars dargestellten Figur von einem phantasmatischen Körper sprechen? Erinnern wir uns, daß Lacan das Phantasma als treffendes Beispiel für das objet a bezeichnet. Das objet $a$, welches als Objekt in der imaginären Spiegelbeziehung auftaucht, hat nicht dem narziBtischen Begehren des Subjekts zu genügen, sondern seinem imaginären Anspruch. Indem es einen Mangel verdeckt, gewährt es Homogenität (so bietet zum Beispiel das Idol eine imaginäre Befriedigung des Mangels).

Anhand des phantasmatischen Körpers, der in Kolmars Text präsentiert wird, zeichnen sich nun folgende Züge des totalitären Paradigmas der Kulturation ab: Es geschieht eine Umwendung des Anderen in das Selbe, innerhalb derer sich ein nicht-identifizierbares Anderes zu einem identifizierbaren Anderen wandelt. Diese Umwendung impliziert eine Tilgung des Anderen im Sinne von Lévinas - also ein Auslöschen der Differenz, welche die Einheit und die Kontinuität des Selbst ursprünglich in Zweifel zieht, indem sie seinen Mangel aufdeckt. Für Lévinas würde dies bedeuten, den Horizont des sozialen oder des intersubjektiven Daseins zu schließen - und hier deutet sich ein Berührungspunkt mit dem theoretischen Modell Lacans an, wenn wir Lévinas' Kategorie des Intersubjektiven (das Für-den Anderen-sein, das Sagen) und Lacans Kategorie des Symbolischen aufeinander beziehen. Das totalitäre Paradigma öffnet also ein Feld des reinen Imaginären.

Die kulturelle Inskription des weiblichen Körpers (die Ver-Körperung des Bösen), welche als schockhaftes Ereignis eintritt, als eine Gewalttat, die den Leib verletzt und eine andauernde Qual bedeutet, folgt der Logik der Bildung eines Phantasmas, das einen stabilisierenden imaginären Anspruch erfüllt: es gewährt eine Identität, die lückenlos und widerspruchsfrei ist. Die fatale Befestigung des Bildes des Bösen und des Schreckens am weiblichen Körper erlaubt dessen Externalisierung, wobei die kulturellen Normen, etwa die Repräsentationsweise des Bösen, bestätigt werden. Der Einbruch der Dimension des Bildlichen in die Sphäre des Leiblichen, die Kondensation von Körper und Bild, die eine Transparenz von Signifikant und Signifikat erzwingt, führt uns in jenen Bereich des Imaginären, in dem es nicht nur um eine Tilgung des Anderen geht, sondern auch um eine Verletzung des Realitätsprinzips. 


\section{Konterkarierung}

Nun bilden die beiden Paradigmen der Kulturation, die ich anhand des phantasmatischen und des decouvrierten weiblichen Körpers zu entwerfen versuchte, keineswegs eine starre Opposition; vielmehr überlagern sie einander. Ein solcher Kreuzungspunkt zeichnet sich etwa dort ab, wo beide Paradigmen eine Transparenz zwischen Körper und Zeichen herstellen - sei es in der Form der organischen Einheit von soma und sema bei der Frau mit dem Teufelshaupt, sei es in der semiotischen EntblöBung der gebärenden Frau. Doch während die damit verbundene Aufgabe an Differenz im Falle der gebärenden Frau die Erfahrung einer Entwürdigung bedeutet, eines momentanen Verlustes an Autorität und Würde, welcher vielleicht eine tiefgreifende Kränkung wiederholt, aber doch den Charakter des Ereignishaften trägt, weist sie im Falle der flüchtenden Frau auf die dauerhaften Spuren einer Traumatisierung hin, die eine endlose Panik auslöst. Der decouvrierte Körper verweist noch immer auf die Kehrseite der Kulturation - auf die Hoffnung, sich durch die Aneignung der Sprachen im sozialen Raum zu verankern. Bei Kolmar scheint diese Kehrseite blind.

Eine solche Deutung der Kulturation sollte aber bezogen werden auf den historischen und politischen Kontext, in dem die beiden Texte, die ich hier diskutiert habe, entstanden sind: im Wien der Jahrhundertwende und in der Weimarer Republik. Auch sollten die beiden Paradigmen der Kulturation in Beziehung gesetzt werden zur jeweiligen Schreibposition, die Kolmar und Freud einnehmen: Kolmar entwirft ihr Selbstbildnis (genauer gesagt, einen Aspekt ihres Selbstbildnisses) durch das totalitäre Paradigma, das heißt mittels und in der Durchquerung dieses Paradigmas. Sie notiert also den zerstörerischen Weg einer Selbst-Identifizierung durch jenes übermächtige Haßbild hindurch, das die umgebende Gesellschaft projiziert. Bei Freud hingegen geht es um die Fundierung der psychoanalytischen Theorie, welche mit dem Paradigma der Assimilation vermittelt erscheint. Eine mögliche Version dieser Vermittlung deutet Sarah Kofman an, wenn sie in der Leugnung der jüdischen Identität, die sie bei Freud vermutet, eine Verallgemeinerung dieser Identität wahrnimmt - eine Verallgemeinerung, um »der ganzen Menschheit (...) mit dieser gefährlichen Gabe ihre sichergeglaubte Identität für immer zu nehmen.« (Kofman 1990, 163) Denn während der phantasmatische Körper gleichsam einen Trug repräsentiert und bestätigt und dadurch eine stabilisierende Wirkung ausübt, ist die Beruhigung, welche der einen Trug aufdeckende decouvrierte Körper gewährt, uneindeutig: für die lachenden Dritten wird er zum Objekt selbstgewisser Belustigung und obszönen Genusses, weil sie als unbeteiligte Zuschauer miterleben, wie ein Betrug auffliegt.

Doch für Freud - und vielleicht für jede analytische Position - wird der decouvrierte Körper zum punctum (Barthes 1985, 53) - das heißt zu jener Stelle, die mehrere Bedeutungsschichten zusammenheftet: der Körper als ein Detail des Abgebildeten, das den Betrachter zugleich anzieht und verwundet. 


\section{Anmerkungen}

1 Ich ziebe hiet bewuBt den Begriff der »Assimilation « gegenüber dem der "Akkulturation « vor, da er die Einseitigkeit der Angleichung stäker betont und eber die Erwartung zum Ausdruck bringt, daB die Differenz, der Mangel, etwas zu Verbergendes sei (wie es Freuds Wendung von det Entlarvung impliziert).

2 Dem Einwand, das Wort "Große Revolution* würde einen eindeutigen Bezug zur Zeit der Französischen Revolution herstellen, möchte ich zum einen die differenzierte Auseinandersetzung Kolmars mit Mythos und Hauptfiguren der Revolution, wie sie sich in ihrem Robespierre-Zyklus niederschlägt, entgegenbalten (Kolmar 1960, 373-457), zum anderen die nationalstaatliche Ordnung, auf welche die vierte Strophe des zitierten Gedichts anspielt.

\section{Literatur}

Barthes, Roland (1985): Die helle Kammer. Bemerkung zur Photographie. Frankfurt/M.

Freud, Sigmund (1982): Der Witz und seine Beziebung zum UnbewuBten. In: Psychologische Schriften. Studienausgabe Band IV., Frankfurt/M.

Kofman, Sarab (1990): Die lachenden Dritten. Freud und der Witz. München, Wien.

Kolmar, Gertrud (1960): Das lyrische Werk. München.

Laplancbe, J., Pontalis, J.-B. (1989): Das Vokabular der Psychoanalyse. FrankfurvM., 9. Auff.

Lévinas, Emmanuel (1983): Die Spur des Arderen. Untersuchungen zur Phänomenologie und Sozialphilosophie. Freiburg, München.

Lévinas, Emmanuel (1986): Ethik und Unendliches. Graz, Wien.

Lévinas, Emmanuel (1987): Totalität und Unendlichkeit. Versuch über die Exteriorität. Freiburg, München.

Scarry, Elaine (1990): Schmerz und Macht. Anatomie der Folter. In: Neue Rundschau 101, Heft 3. Waroer, Marina (1985): Monuments and Maidens. The Allegory of the Female Form. London. Zizek. Slavoj (1989): The Sublime Object of Ideology. London, New York.

Zizek, Slavoj (1991): Rossellini: Die Frau als Symptom des Mannes. In: Lettre International. Nr. 12 , S. 80-88. 